\title{
Accident Detection, Avoidance and Prevention using Intelligent Transportation System
}

\author{
Somya Sharma \\ Associate Lecturer \\ Shobhit University,Meerut
}

\author{
Rajesh Pandey \\ Assistant Professor \\ Shobhit University,Meerut
}

\begin{abstract}
India is a nation which has the one of the largest Non-Lane based road network in the world. Developed Countries nowadays uses Intelligent Transportation system (ITS) to solve traffic related problems and provide smooth and safe ride on roads. The road traffic congestions are recurring problem in India due to the poor infrastructure, attitude of road users in India and daily increase in number of vehicles. So to overcome this issue VANET [Vehicular Ad Hoc Network] has come with lot of ideas such as vehicular communication, traffic controlling, Navigation and other application in VANET. Through this review paper, it has been tried to find out an innovative approach as VANETs in solving traffic problems that has significantly large amount of public and private vehicles on roads.
\end{abstract}

\section{Keywords}

Accident Detection, Intelligent Transportation System

\section{INTRODUCTION}

In an ITS (Intelligent transportation systems), VANETs form a key component. It works on the basics of MANETs (mobile ad hoc networks). MANETs are considered as a self network. That is, the network is formed by itself; hence they do not require any centralized control mechanism. Hence, in the ad hoc network concept each node can act as both a router as well as a data terminal. VANET is part of MANET, this way that all node can move liberally surrounded by the network reporting and wait linked, all node can talk by way of additional nodes in only hop or multi hop, and a few node could be Vehicle, Road Side Unit (RSU). The main objectives of this task are to answer mechanical issues correlated to communication protocols as well as data safety for car-to-car exchanges. The ultimate challenge was to solve the trouble of how to contact nodes not straight within data lines collection by employ neighbors as forwarders. [1].

In [2] the authors have proposed an efficient conditional privacy preservation (ECPP) protocol for safe vehicular interactions. The ECPP protocol can powerfully contract with the increasing revocation file even as achieving conditional traceability by the authorities. Instead of relying on a huge storage space at each OBU-On board Unit, as most of the previously reported schemes did, the future protocol be able to keep the necessary unknown key cargo space minimal without losing the security level. Meanwhile, the future protocol gain qualities in the fast authentication on safety messages and an efficient

Conditional privacy track means, which can give out as an brilliant applicant for the future VANETs.

\section{LITERATURE REVIEW}

In 2007, Fussler, H., discussed the history of vehicular ad-hoc networks and shows the early vision of creating a huge MANET that would facilitate cheap and ubiquitous communication on the ISM band, and how this vision was reduced to cross sending emergency information in a geographically limited area.

Leung, K. K., proposed the concept of node connectivity in vehicular ad-hoc networks. He focused on studying transport system with structured mobility. He provides an analytical framework including the design requirements of the mobility model for realistic vehicular networks

In 2013, Pandit, K. and Ghosal, D., proposed to use vehicular ad hoc networks (VANET) to collect and aggregate real time speed and position information on individual vehicles to optimize signal control at traffic intersections. They give an online algorithm, referred to as the oldest job first (OJF) algorithm, to minimize the delay across the intersection.

Sok-Ian sou gives brilliant idea about modelling Emergency messaging for car accident over Dichotomized headway model. This paper proposes an analytical model for evaluating the performance of emergency messaging via wireless CA systems. He utilizes the dichotomized headway model, the braking model, and Greenberg's logarithmic model to generate vehicular mobility traces for analysis.

In 2014, Penna, K., proposed evaluation of active position detection in vehicular Ad-hoc networks. The main contribution of their approach by running an ns- 2 simulation with dynamic number of nodes in various mobility scenarios such as urban, rural, Manhattan. Their first proposed algorithm replies on signal propagation time for verifying the position. And their second algorithm verifies the position information with the help of base station located in the coverage area of the vehicular networks.

\section{INTELLIGENT TRANSPORT SYSTEM}

In an ITS (Intelligent transportation systems), VANETs form a key component. It works on the basics of MANETs (mobile ad hoc networks). MANETs are considered as a self-network. That is, the network is formed by itself, hence they do not required any centralized control mechanism. Hence, in the ad hoc network concept each node can act as both a router as well as a data terminal. VANET is part of MANET, this way that all node can move liberally surrounded by the network reporting and wait linked, all node can talk by way of additional nodes in only hop or multi hop, and a few node could be Vehicle, Road Side Unit (RSU). The main objectives of this task are to answer mechanical issues correlated to communication protocols as well as data safety for car-to-car exchanges.

\subsection{Types of communication}

The possible types of communications:

3.1.1 Vehicle To Vehicle (V2V): It provide interaction within vehicles in ad hoc approach. In V2V, a vehicle can accept broadcast and exchange helpful traffic news i.e., traffic 
conditions and road accidents in particular area or with other vehicles.

3.1.2 Vehicle to Infrastructure (V2I): In this communication type, the information will be broadcast between the nodes (i.e vehicle) and the infrastructure (said as ITS), to discuss about valuable information such as road conditions and safety events which have been taken into account. In this V2I, a vehicle (node) launches a connection between RSU and contact with external networks which is internet.

\section{VANET}

Vehicular Ad-Hoc Network or VANET is a technology that has moving vehicles as nodes in a network for creating a mobile network. We can say that VANET turns each and every vehicle into a wireless node, allowing cars to connect to each other which are 100-300 meters apart and, in turn, create a wide range of network. As cars fall out due to signal range and drop out of the present network, other cars can join in to connect vehicles to one another so a mobile Internet can be created. Vehicular networks are very fast emerging for deploying and developing new and traditional applications. It is characterized by rapidly changing topology, high mobility, and ephemeral, one-time interactions. Both MANETs and VANETs are characterized from the movement and selforganization of the nodes.

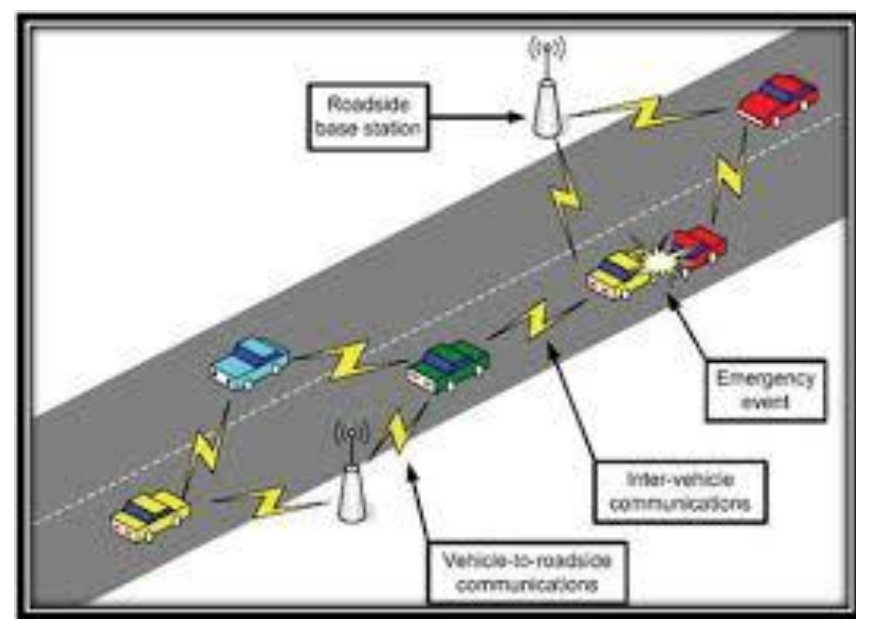

\subsection{Smart Vehicles}

Vehicles which are equipped with multi interface cards and sensors, on board unit and externally. The number of vehicles equipped with on-board wireless devices (e.g., UMTS, IEEE 802.11p, Bluetooth, etc.) and sensors (e.g., radar, ladder, etc.), is increasing for efficient transport and management applications are focused on optimizing flows of vehicles by reducing the time taken to travel and avoiding any traffic congestions. As an instance, the radar present on on-board could be used to sense traffic congestions and automatically slow the vehicle. In another accident warning systems, sensors can be used to determine that a crash may be occurred if air bags were deployed; this kind of information is then relayed via V2I or V2V within the vehicular network. A provide different levels of functionality is provided by using number of systems and sensors. The major systems and sensors exploited for intra-vehicle communications we cite: crash sensors, the data recorder, the braking system, the engine control unit, the electronic stability control, the infotainment system, the integrated starter generator, the electronic steering, the tire pressure monitoring system, the power distribution and connectivity, the lighting system, seat belt sensors, etc.
Commonly, a smart vehicle is equipped with the following technologies and devices:

(i) A wireless transceiver for data transmissions among vehicles $(\mathrm{V} 2 \mathrm{~V})$ and from vehicles to RSUs (V2I);

(ii) A Central Processing Unit (CPU) which implements the applications and communication protocols;

(iii) A Global Positioning Service (GPS) receiver for navigation and positioning services;

(iv) An input/output interface for the interaction of human with the system;

\section{WORKING}

The ESPM framework performs prediction of road accident in three steps:

(i) the RSU receives periodical Status Report (SR) from the vehicles in its range,

(ii) the RSU receives Traffic Flow (TF) data from the highway road, and

(iii) the RSU performs prediction of road accident based on traffic flow analysis.

\subsection{Reporting Phase}

In the reporting phase, once a vehicle enters into the coverage area of the RSU, it reports about its status to the RSU periodically in the form of a Status Report (SR)

\subsection{Monitoring Phase}

In this phase, the RSU performs traffic flow monitoring based on the traffic flow data. The traffic flow data can be obtained with the help of sensors embedded in the four lane highway road called Four Lane Sensor Grid (FLSG).

\subsection{Prediction Phase}

This phase is instrumental for predicting emergency situation based on the SR and TF data received from reporting and monitoring phases respectively. Between SR and TF values, if either one is abnormal, then this phase alarms by predicting an emergency situation (i.e. the possibility of occurrence of an accident). This phase is categorized into three modules:-

- first module is used to assess the abnormality based on the status report of the vehicle,

- $\quad$ second module is used to assess the abnormality based on the traffic flow in the highway road and

- third module is used to compute prediction based on first and second modules.

In ESPM, the prediction of emergency situation is done by the Road Side Unit (RSU) based on

the Status Report (SR) send by the vehicles in its range and the Traffic Flow (TF) analysis done in the highway road.

Once the emergency situation is predicted in advance, it will be possible to initiate a prevention process by alerting vehicles to avoid highway road traffic accidents. This can be accomplished by constructing and disseminating Emergency Warning Message (EWM) to all vehicles in the coverage area of the RSU and to the near-by RSUs. ESPM performs well in emergency situation prediction before the occurrence of an accident in a proactive manner. ESPM predicts the 
emergency situation within 0.20 seconds, which is comparatively lesser than the statistical value. The prediction accuracy of ESPM with respect to vehicle density is found to be 92 percent in different road traffic scenarios.

\section{CONCLUSION}

An Emergency Situation Prediction Mechanism (ESPM) framework is developed and validated to predict the possibility of occurrence of an accident in Indian four lane highway road. The primary objective of ESPM is to predict an emergency situation in a proactive manner. Hence it attempts to prevent road accidents so as to reduce the death toll and injury rates. ESPM performs prediction of an emergency situation in three phases such as reporting, monitoring and prediction.

\section{REFERENCES}

[1] Ghassan Samara, wafaa. A. H, Al-Salihy, R. Sures, "Security Issues and Challenges of Vehicular Ad Hoc Networks (VANET)", IEEExplore.

[2] RongxingLu, XiaodongLin, HaojinZhu, Pin-HanHo and Xuemin (Sherman) Shen, " ECPP: Efficient Conditional Privacy Preservation Protocol for Secure Vehicular Communications", IEEE Explore

[3] Kumar, A., Sinha, M., "Overview on Vehicular ad hoc network \& its security issues", computing for sustainable
Global Development (INDIACom) 2014 International Conference on, vol., no., pp. 792, 797, 5-7 march 2014.

[4] Penna, K., Yalavarthi, V., Huirong Fu Zhu, "Evaluation of active position detection in vehicular Ad-hoc Networks", Neural Networks (IJCNN), 2014 International Joint Conference on, vol. no. , pp. 2234, 2239, 6-11 July 2014

[5] Fussler, H., Schnaufer, S., Transier, M., Effelsberg, W., "Vehicular ad-hoc networks: from vision to reality and back", wireless on Demand Network Systems and Services, 2007. WONS'07. Fourth Annual Conference on, vol., no., pp.80,83, 24-26 Jan. 2007

[6] Pandit, K., Ghosal, D., Zhang, H. M., Chen-Nee Chuah, "Adaptive Traffic signal control with vehicular Ad-hoc Networks", Vehicular Technology, IEEE Transactions on, vol. 62, no.4, pp. 1459,1471, May 2013

[7] Sok-Ian Sou, "Modeling Emergency messaging for carAccident over Dichotomized Headway Model in Vehicular Ad-hoc Networks", communications, IEEE Transaction on, vol 61, no.2, pp.802, 812, February 2013

[8] Ho, I. W. H., Leung, K. K., "Node connectivity in vehicular Ad Hoc Networks with Structured Mobility", Local computer Networks, 2007. LCN 2007. 32nd IEEE conference on, vol., no., pp. 635,642, 15-18 Oct. 2007. 